\title{
SUR L'UTILISATION DES SÉRUIMS DE CASÉINE ET DES PETITS LAITS DE FROMAGERIE
}

\author{
par M. BEAU. \\ Ingénieur agronome.
}

Les industries fromagère et caséinière laissent toutes deux, après coagulasion de la caséine, un résidu presque limpide, de couleur jaune verdâtre, que l'on appelle (1) petit lait dans le cas des fromages et sérum dans le cas de la caséine, et auquel on, peut attribuer en moyenne la composition suivante en grammes par litre:

\begin{tabular}{|c|c|}
\hline Densité . . . . & 1,025 à 1, \\
\hline $\begin{array}{l}\text { Lactose (plus ou moins partiellement transformé } \\
\text { en acide lactique) }\end{array}$ & 50 \\
\hline $\begin{array}{l}\text { Matières azotées (constituées presque exchasivenent } \\
\text { par de l'albumine) }\end{array}$ & 10 \\
\hline $\begin{array}{l}\text { Sels minéraux (en majeure partie solubles ou } \\
\text { solubilisés) }\end{array}$ & \\
\hline total & \\
\hline
\end{tabular}

soit en moyenne 65 grammes de matières sèches par litre, dont 60 grammes de matières alimentaires entièrement digestibles.

C'est cette valeur alimentaire du sérum qui a conduit tout naturellement, au début des industries fromagère et caséïnière, ì donner ce produit aux porcs, qui seuls ont été jusqualors capables de l'utiliser comme aliment. La caractéristique capitale de ce produit est en effet sa dilution considérable, sa teneur en eau atteignant 93 à $94 \%$. Seul le tube digestif du pore a une constitution lui permettant de supporter une pareille dilution. Aucun autre animal domestique n'est capable d'abscrber ce produit et les essais tentés à cet égard avec les chevaux et les bovidés n'ont donné aucun résultat. En particulier, l'acidité toujours élevée du produit semble être un obstacle absolu à la chose, sans compter les fermentations secondaires qui accompagnent toujours la prcduction de l'acide lactique.

Quoiqu'il en soit, l'utilisation alimentaire des sérums et petits laits par les porcs a pris depuis le début du $\mathrm{XX}^{\mathrm{e}}$ siècle une importance croissante, par suite du développement également croissant de la production fromagère et caséinière. En particulier, les quantités de lait traitées dans un même centre étant de plus en plus considérables, et atteignant dans un nombre de cas de plus en plus grand, plusieurs dizaines de milliers de litres par jour, on a été amené à annexer aux laiteries des porcheries de plus en plus importantes. Déjà avant la guerre, on trouvait dans les Charentes et en Normandie des porcheries avec un

(I) Au contraire de ce qui se passe en France, les pays anglo-saxons et germano-scandinaves n'ont qu'un seul mot pour désigner ce produit: whey en anglais, vei en hollandais, molke en allemand, valle en scandinave. 
effectif dépassant 500 têtes, ce qui, à raison de 40 litres de sérum par jour, ne correspond encore $q u$ 'à l'utilisation journalière d'une vingtaine de mille litres de petit lait.

Or, on trouve actuellement, surtout en Normandie, réunies en un seul point, des quantités de sérum dépassant ces chiffres, pouvant atteindre jusqu'à 50.000 litres par jour. L'établissement d'une porcherie susceptible d'absorber une pareille quantité de liquide, e'est-à-dire nécessitant un effectif de plus de 1.000 porcs, devient une opération extrêmement dangereuse, d'une part à cause des épidémies toujours possibles, - on peut même dire toujours certaines, la science vétérinaire ne permettant pas de garantir la santé d'un pareil effectif dans tous les cas,- d'autre part à cause de l'énorme mise de fonds que représente ledit effectif (1,000 pores pesant 100 kilos représentent actuellement une valeur comprise entre un demi million et un million de francs, auxquels il faut ajouter la valeur des constructions et installations), En cas d'épidémie en particulier la perte porte non seulement sur le sérum consommé, mais aussi sur la valeur du cheptel.

La très grande porcherie est done un gros danger, et comme il n'est guère possible de transporter le sérum, vu sa valeur extrêmement faible due à sa grande dilution, on ne peut songer à répartir ce dernier dans plusieurs porcheries suffisamment éloignées l'une de l'autre pour pallier aux risques de contamination. Ajoutons à cela que les quantités de petit lait varient considérablement d'une saison à l'autre, ce qui nécessite une variation correspondante du cheptel, et qu'enfin les prix de vente des pores varient dans de telles proportions qu'on n'est jamais certain, quand on achète un lot de pores à engraisser, de retrouver l'argent dépensé pour l'achat, trois ou six mois après, lors de la revente.

De tout cela il est facile de conclure que la porcherie est une quasi impossibilité pour les grosses laiteries et que seule une utilisation industrielle devra permettre de résoudre le problème.

Théoriquement les Utilisations industrielles des petits laits et sérums sont assez nombreuses pour qu'il semble qu'on n'ait que l'embarras du choix, fabrication du lactose, de l'acide lactique, du vinaigre de lait etc... Pratiquement, il n'en est malheureusement rien. Toutes ces utilisations industrielles sont en effet basées exclusivement sur la présence du lactose ou sucre de lait dans les produits en question. II n'a jamais été tenté industriellement d'extraire la lactalbumine, qui n'a du reste jusqu'alors pas d'emploi sérieux et régulier. D'ailleurs la majeure partie de l'extrait sec est constitué par le lactose, et, de même qu'on a successivement tiré du lait le beurre, puis la caséine, il semble logique d'en extraire le lactose.

En pratique cette Extraction du lactose se heurte à des difficultés considérables: on premier lieu la déplorable facilité avec laquelle le lactose se transforme spontanément sous l'influence des ferments 
lactiques en acide lactique (dans la fabrication de la caséine lactique, la production de cet acide est même la base de la précipitation de cette dernière) fait que, d'une part, une partie du lactose disparaît et que d'autre part, l'acidité du produit nuit à la fabrication, nécessite l'addition d'un neutralisant minéral qui charge les liqueurs en cendres, alors que ie produit final doit en être entièrement exempt : bref ce n'est guère qu'avec des sérums très frais, tels que ceux de caséine précipitée de laits sans acidité, au moyen de présure ou de très petites quantités d'acide sulfurique, ou encore avec des sérums de fromages à pâte dure (Gruyère), préparés également par la présure et avec des laits très frais, que l'on a pu jusqu'alors fabriquer économiquement le lactose. Si l'on songe qu'en France, la production fromagère comprend surtout des fromages à pâte molle à petits laits acides, et que la production caséinière, surtout dans la moitié nord du pays, est en majeure partie une production de caséine lactique, on comprend facilement que la lactoserie ne peut résoudre le problème posé.

Ajoutons à cela que le matériel nécessaire à cette fabrication est très coûteux; que la fabrication elle-même est délicate et dominée par la nécessité d'obtenir un produit chimiquement pur, exempt notamment d'albumine et de cendres, entin que le lactose n'a, au contraire de la caséine, qu'un débouché fort restreint, notamment en pharmacie et en confiserie, et l'on comprendra encore mieux que cette industrie ne puisse résoudre la difficulté. En fait les usines de lactose sont en France très peu nombreuses; deux seulement sont en fonctionnement, celle de Chef-du-Pont (Manche), et celle de Sains-du-Nord (Nord). Trois autres fondées pendant ou un peu avant la guerre n'ont donné aucun résultat et ont dû être liquidées avec grosses pertes ; ce sont les usines de Courçond'Aunis (Charente-Inférieure), de Sottevast (Manche), et d'Orbec (Calvados).

A l'étranger, les lactoseries sont plus nombreuses et surtout de dates plus anciennes, et par suite grevées de frais d'établissement moindres ; elles se sont principalement développées dans certains pays à climat froid, où les laits ont peu d'acidité, et sont principalement transformés en fromages à pâte dure, notamment en Allemagne, en Hollande et dans le nord de l'Amérique. De là résulte aussi que ce sont les trois pays qui, par les quantités produites, dominent le marché, produisant des variations de cours parfois catastrophiques, et rendent cette industrie encore plus difficile à conduire, malgré la faible valeur de la matière première.

La production française annuelle de lactose (1) atteint environ 700 tonnes, ce qui représente un peu plus de 70 millions de litres de sérum. Il s'agit là exclusivement de lactose Codex, très raffiné, se dissolvant

(1) Nous devons les chiffres suivants à l'amabilité de M. Lizé, Président du Syndicat des Fabricants de Sous-produits et Dérivés du Lait, à qui nous adressons nos remerciements à cette occasion. 
entièrement dans J'eau sans résidu et ne Iaissant rien après calcination. Le marché de ce produit est du reste mondial, et les prix intérieurs sont entièrement conditionnés par les prix extérieurs avec un droit d'entrée qui atteint actuellement $1 \mathrm{fr}, 20$ le kilogramme. La moitié de la production est exportée.

La Fabrication de l'acide lactique, qui semblerait théoriquement être la plus facile à mener ainsi que la plus logique, étant donné la transformation abondante du lactose en acide lactique (1), n'a jusqu'alors rien donné dans la pratique. Déjà avant la guerre, la fabrication de l'acide lastique en partant du riz était plus économique et donnait un produit plus pur qu'en partant du petit lait. Un essai industriel en grand tenté récemment au Molay-Littry (Calvados) a confirmé cette conclusion, l'exploitation ayant dû être arrêtée après quelques années de fonctionnement. On ne peut en pratique compter sur cette industrie pour utiliser les sérums disponibles.

Il en est de même de la Fabrication du Vinaigre de lait. Celle-ci consiste à transformer par deux fermentations successives le lactose en alcool au moyen de levures spéciales, puis ce dernier en acide acétique au moyen du Mycoderma aćti habituel (2). Au point de vue technique, la première difficulté consiste à éviter la fermentation lactique qui est déjà toujours amorcée dans les sérums et tend toujours ì reprendre, même après pasteurisation. De plus, le produit obtenu a presque toujours un gont de petit lait, auquel le consommateur s'habitue difficilement ; là encore il ne semble pas que cette fabrication puisse remplacer celle du vinaigre de vin. En fait la seule et unique usine ayant essayé industriellement cette préparation et qui était avant la guerre installée à Condac (Charente), a dû rapidement fermer ses portes par suite du du manque de débouchés.

En résumé, en dehors de l'industrie du lactose, aucune autre utilisationation industrielle ne permet jusqu'alors de traiter les petits laits et sérums disponibles. Mais la lactoserie est loin de pouvoir utiliser les grosses quantités existantes, et il n'y a en outre aucun intérêt à augmenter la quantité de lactose sur le marché, toute quantité supplémentaire devant amener un effrondrement des cours déjà très bas. II faut donc cheercher autre chose pour résoudre le problème des sérums.

En somme la grosse difficulté provient de ce qu'on se trouve en présence d'un produit très diluś, impossible à conserver, et dont le

(1) Suivant la formule: $\begin{gathered}\mathrm{C}^{12} \mathrm{H}^{12} \mathrm{O}^{11} \\ \text { Lactose }\end{gathered}+\underset{\text { Eau }}{\mathrm{H}^{2} \mathrm{O}}=\underset{\text { Acide lactique }}{4 \mathrm{C}^{4} \mathrm{H}^{6} \mathrm{O}^{3}}$

(2) La transformation se fait selon les formules suivantes: $\mathrm{C}^{12} \mathrm{H}^{22} \mathrm{O}^{11}+\mathrm{H}^{2} \mathrm{O}=4 \mathrm{C}^{2} \mathrm{H}^{6} \mathrm{O}+4 \mathrm{CO}^{2}$ lactose eau alcool acide carbonique $4 \mathrm{C}^{2} \mathrm{H}^{6} \mathrm{O}+\mathrm{O}^{4}=4 \mathrm{C}^{2} \mathrm{H}^{4} \mathrm{O}^{2}$ alcool oxygène acide acétique 
principal composant, le lactose, est très difficile à extraire et n’a que des débouchés très restreints.

Mais l'ensemble de la matière premiềre n'en constitue pas moins, y compris les matières autres que le lactose qu'elle renferme, notamment l'albumine, une matière alimentaire ayant une certaine valeur, au moins pour les animaux, puisqu'en particulier elle est dans bien des cas donnée aux pores.

Il était done logique de chercher dans la voie de l'alimentation du bétail une nouvelle utilisation des sérums, la question des débouchés ne paraissant du reste pas se poser, vu l'étendue des cheptels, et la grosse consommation qu'ils font de matières alimentaires, Mais pour résoudre le problème, il faut supprimer les deux difficultés indiquées ci-dessus, c'est-ì-dire en faire un produit concentré et conservable.

La concentration coûte nécessairement toujours cher, si l'on songe que les sérums renferment de 93 à $94 \%$ d'eau et toute la difficulté du problème consiste à évaporer cette eau avec le minimum de vapeur, c'est-à-dire de charbon. On sait que un kilogramme de bon charbon donne huit kilogrammes de vapeur d'eau, susceptibles d'évaporer théoriquement huit kilogrammes d'eau et pratiquement 6 à 7 kilogrammes seulement.

La conservabilité d'autre part ne peut être obtenue pratiquement d'une façon commode qu'en transformant le sérum liquide en un produit solide pratiquement sec, e'est-à-dire ne renfermant pas plus de $10 \%$ d'eau.

Si nous prenons la composition du sérum indiquée au début de cette étude, on voit qu'il faut réduire 100 litres de sérum à environ 7 kilos de produit pratiquement sec, soit une évaporation de 93 kilos d'eau, correspondant à une dépense de 14 kilos de charbon. Pour obtenir 100 kilos de produit sec, il faudrait done 200 kilos de charbon valant actuellement $30 \mathrm{fr}$. en chiffres ronds : c'est là une dépense très élevée à la quelle il faut encore ajouter tous les autres frais; quant au prix de vente il ne peut évidemment dépasser celui des aliments concentrés d'une valeur nutritive égale. Or le son vaut actuellement $80 \mathrm{fr}$. les cent kilos et l'orge de mouture $100 \mathrm{fr}$.

Les premiers essais que nous avons faits à cet égard datent de 1919, aussitôt après la guerre; ils nous avaient déjà donné à cette époque un produit acceptable, mais la grande question restait toujours celle de l'économie de combustible pour l'évaporation, et ce n'est que peu à peu que la difficulté a pu être résolue. Nous avons d'abord songé à utiliser les appareils d'évaporation tubulaires, à simple ou multiple effet, avec ou sans thermocompression; malheureusement, outre le prix extrêmement élevé de ces appareils, leur complication et leur construction tubulaire elle-même, les rend à peu près impropres à ce genre d'opérations : en particulier, lorsque l'on doit, comme c'est le cas, pousser la 
concentration très loin, on ne peut entièrement éviter les gratinages et incrustations dans les tubes, d'où des difficultés prohibitives de nettoyage. De ce fait ces appareils sont le plus souvent très délicats à conduire, et leur emploi est sinon hors de question dans cette affaire, tout au moins ne peut se faire que dans des conditions très particulières.

Quant à l'économie de charbon, c'est par une utilisation judicieuse des échappements que l'on peut abaisser la consommation de ce dernier jusqu'à la moitić environ de la quantité théorique, si bien que cette dépense n'entre plus dans le prix de revient que d'une manière acceptable.

On obtient ainsi un sirop de 1.300 de densité, renfermant quelque $50 \%$ d'extrait sec. Ce sirop peut être traité en vue de donner un produit ne renfermant plus que $10 \%$ d'eau, c'est-à-dire pratiquement aussi facile à conserver que les farines, issues ou déchets employés à l'alimentation du bétail. Un produit de ce genre peut être obtenu par dessiccation du sirop, soit directement en prenant les précautions nécessaires pour éviter une trop forte caramélisation pouvant facilement passer à la carbonisation des matières organiques, soit indirectement par mélange avec une substance appropriée et dessiccation indirecte subséquente. Il faut seulement que l'on n'ait pas besoin d'ajouter une trop grande quantité de cette dernière, auquel cas d'autres difficultés économiques pourraient surgir du fait du prix de la substance en question.

On obtient ainsi une sorte de farine grossière, que nous avons appelée Lactosine (1), en souvenir de son origine lactée, et de sa richesse en lactose, de couleur variant du blond au brun clair, et dont la composition est sensiblement la suivante en movenne, comparée à celle de la farine d'orge :

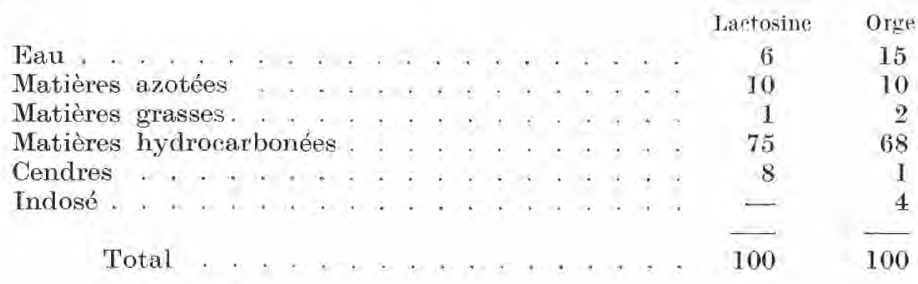

Comme on le voit la lactosine a une valeur nutritive très analogue à celle de la farine d'orge, tout en coûtant très notablement moins cher.

La lactosine se donne à tous les animaux de la ferme : les chevaux la prennent très bien telle quelle, après humectation préalable avec un peu d'eau. Les animaux d'engrais ou de lait, boufs, vaches, moutons et pores la prennent également humidifiée ou en cuvées ou mélangées à d'autres aliments plus aqueux, tels que betteraves, pommes de terre, tourteaux, etc...

Du reste, il est possible dans le cours de la fabrication de faire varier

(1) Nom déposé selon la loi. 
la teneur en matières albuminoïdes du produit, de façon à préparer des lactosines plus ou moins riches en azote suivant le but que l'on se propose.

\title{
L'ALLAITEMENT ARTIFICIEL CHEZ LES ANIMIUX DOMIESTIQUES
}

\author{
Par R. BUS, \\ Docteur-Vétérinaire
}

L'étude de l'allaitement artificiel constitue un grand chapitre de la pathologie humaine, mais elle est moins importante en médecine vétérinaire. Les animaux soumis à l'allaitement artificiel sont plutôt des exceptions.

Si l'allaitement artificiel ne conduit pour ainsi dire jamais à des échecs chez les animaux, il offre, cependant, certaines difficultés, lesquelles arrêtent très souvent l'éleveur. Pour celui-ci, le jeune est, avant tout, une source de profits, aussi les principes qui dirigentl'animaliculteur sont-ils un peu opposés à ceux qui animent le puériculteur.

L'allaitement artificiel est homologue ou hétérologue; dans le premier cas, le lait que recevra le jeune appartient à la même espèce que lui ; dans le second cas, à une espèce différente. Il faut également envisager le cas où le lait sera puisé directement à la mamelle, que la mère soit de la même espèce ou non que le nourrisson, et celui où le lait sera donné au biberon, alors même qu'il proviendra de la même espèce que le jeune.

Lorsque 1'on soumet un jeune animal d'une espèce quelconque à l'allaitement artificiel, on constate, règle générale, que dans $95 \%$ des cas ce procédé réussit, en supposant bien entendu que les règles d'hygiène les plus élémentaires soient suivies. L'allaitement artificiel a échoué lorsque les conditions hygiéniques étaient déplorables. Il fallait s'y attendre. Les détracteurs de ce mode d'alimentation du jeune âge s'appuient sur ce fait, qu'il provoque la diarrhée et prédispose aux infections. Il est bien évident que de tels arguments ont trait, non à l'allaitement artificiel, mais à la façon dont on l'applique. Sans doute, nous ne voulons pas dire qu'il soit supérieur à l'allaitement maternel, ce serait aller contre la nature - , mais nous conseillons à l'éleveur d'y recourir lorsque l'allaitement maternel est impossible, dans les cas de mort des mères, d'anomalies de conformation, d'insuffisance mammaire. Si l'allaitement artificiel ne devait pas avoir des résultats plus brillants qu'en médecine humaine, il n'y aurait aucun intérêt à l'appliquer en vétérinaire. Les animaux mourraient, ou bien, élevés péniblement ils constitueraient par la suite des non valeurs. Ce serait prendre beaucoup de peine pour aboutir à un médiocre résultat. En conséquence, ce serait une mauvaise affaire. 